\title{
Ultra-sensitive chemiluminescence imaging DNA hybridization method in the detection of mosquito-borne viruses and parasites
}

\author{
Yingjie Zhang ${ }^{1,3+}$, Qiqi Liu ${ }^{1,2+}$, Biao Zhou ${ }^{1,2}$, Xiaobo Wang ${ }^{3}$, Suhong Chen ${ }^{1,2^{*}}$ and Shengqi Wang ${ }^{1,2^{*}}$
}

\begin{abstract}
Background: Mosquito-borne viruses (MBVs) and parasites (MBPs) are transmitted through hematophagous arthropods-mosquitoes to homoiothermous vertebrates. This study aims at developing a detection method to monitor the spread of mosquito-borne diseases to new areas and diagnose the infections caused by MBVs and MBPs.

Methods: In this assay, an ultra-sensitive chemiluminescence $(\mathrm{CL})$ detection method was developed and used to simultaneously detect 19 common MBVs and MBPs. In vitro transcript RNA, virus-like particles (VLPS), and plasmids were established as positive or limit of detection (LOD) reference materials.

Results: MBVs and MBPs could be genotyped with high sensitivity and specificity. The cut-off values of probes were calculated. The absolute LODs of this strategy to detect serially diluted in vitro transcribed RNAs of MBVs and serially diluted plasmids of MBPs were $10^{2}-10^{3}$ copies/ $\mu$ and $10^{1}-10^{2}$ copies $/ \mu l$, respectively. Further, the LOD of detecting a strain of pre-quantified JEV was $10^{1.8}-10^{0.8} \mathrm{PFU} / \mathrm{ml}$, fitted well in a linear regression model (coefficient of determination $=0.9678$ ).

Conclusions: Ultra-sensitive CL imaging DNA hybridization was developed and could simultaneously detect various MBVs and MBPs. The method described here has the potential to provide considerable labor savings due to its ability to screen for 19 mosquito-borne pathogens simultaneously.
\end{abstract}

Keywords: Mosquito-borne viruses, Mosquito-borne parasites, Chemiluminescence, DNA hybridization

\section{Background}

Mosquito-borne viruses (MBVs) are transmitted from hematophagous arthropod/mosquitoes to homoiothermous vertebrates. As competent hosts, arthropods can support large virus inocula, and transmit them from an infected donor to a recipient during blood-feeding. Three major families in MBVs include the Togaviridae, Flaviviridae, and Bunyaviridae [1]. The globally pandemic MBV species encompass dengue virus (DENV), Japanese encephalitis virus (JEV), yellow fever virus (YFV), West Nile virus (WNV) [2] and chikungunya virus (CHIKV) [3]. In China, MBVs severely affect people living in tropical regions and rural areas. There

\footnotetext{
* Correspondence: doudoucsh@sina.com; sqwang@bmi.ac.cn

${ }^{\dagger}$ Equal contributors

${ }^{1}$ Beijing Institute of Radiation Medicine, Beijing 100850, People's Republic of China

Full list of author information is available at the end of the article
}

are more than 70 identified flaviviruses, of which 35 can affect humans [4]. Factors like global warming, increased population mobility, and urbanization influence the epidemic scope of MBVs and sometimes even result in sudden outbreak [5]. In 1999, the outbreak of WNV in New York resulted in mass-scale animal mortality [6]. In 2012, the outbreak of WNV in Texas resulted in 1,868 human infections (89 deaths) and an economic cost of more than $\$ 47$ million [7]. In 2010, the outbreak of CHIKV in Guangdong province of China led to 173 cases of human infections [8]. From 2005 to 2006, more than 1.3 million cases of CHIKV infections were reported in India [9]. The infected cases of DENV recorded by the World Health Organization (WHO) were approximately 0.9 million [10]. However, actual infected cases worldwide are likely to be more than 390 million; 96 million infected patients show the clinical or sub- 
clinical symptoms of DENV infection [11]. Each year, more than 20,000 casualties are reported due to severe infections of DENV [12].

In addition, malaria parasites and lymphatic filariae, borne by mosquitoes, are currently the most common parasites in the world $[13,14]$. Malaria and lymphatic filariasis have been considered as the world's highest priority control and eradication parasitic diseases due to their wide prevalence $[15,16]$. In tropical regions, these two parasites share the same propagation host and may also co-infect humans [17]. Mosquito-borne parasites (MBPs) infecting humans include Plasmodium vivax, Plasmodium malariae, Plasmodium falciparum and Plasmodium ovale. Malaria is rampant in Asia, Africa, and Latin America, with half of the world's population living in malaria-risk areas. In 2006, 250 million people were infected by malaria and approximately 1 million deaths were reported, among which children $(<5$ years $)$ and pregnant women were most affected [18]. Mosquitoborne lymphatic filariae include Wuchereria bancrofti, Brugia malayi and Brugia timori. Approximately 1.3 billion people are living in the lymphatic filariasis-risk areas. Following malaria, lymphatic filariasis is the second most prevalent arthropod-borne disease worldwide, which affects 128 million people each year in Asia, Africa, Western Pacific, and parts of America [19].

The spread of the mosquito-borne pathogens from the epidemic areas to new ones poses a serious threat to the monitoring, prevention, and control of disease [20]. Efficient prevention, control, and treatment of mosquitoborne diseases rely on the rapid detection of the mosquito-borne pathogens and accurate diagnosis. Currently, methods for detecting MBVs and MBPs are mainly based on genetic assays, which include real-time PCR [21-25], isothermal amplification [26], oligonucleotide microarray [27] and sequencing [28]. However, these methods were designed for detecting only certain species of MBVs or MBPs. A method that can simultaneously detect common MBVs and MBPs has not yet been reported. In this assay, a reliable and portable, ultra-sensitive chemiluminescence (CL) imaging DNA hybridization was developed and used to simultaneously detect common MBVs and MBPs. In vitro transcript RNA, virus-like particles, and plasmids were established as positive or limit of detection (LOD) reference materials. The specificity and sensitivity of the method was validated in cultivated MBVs, simulated mosquito samples, reference materials, and non-MBVs.

\section{Methods}

\section{Specimen collection and processing}

Cultivated MBVs strains were obtained from the Institute of Microbiology Epidemiology of the Academy of Military Medical Sciences and National Institute for
Viral Disease Control and Prevention, China CDC. Lysates (TIANamp Virus RNA Kit, Tiangen Biotech Beijing Co., Ltd.) were added to all the viral samples prior to experiments. Total RNAs were extracted using TIANamp Virus RNA Kit as described in the manufacturer's protocol (Tiangen Biotech Beijing, China) and stored at $-70^{\circ}$ $\mathrm{C}$ until use. Uninfected Aedes vigilax were obtained from the Institute of Microbiology Epidemiology of Academy of Military Medical Sciences and stored at $-70{ }^{\circ} \mathrm{C}$ until use.

\section{Primers and probes design and evaluation}

In this assay, $12 \mathrm{MBVs}$ and $7 \mathrm{MBPs}$ were chosen for detection: DENV1-4, JEV, YFV, WNV, St. Louis encephalitis virus (SLEV), CHIKV, eastern equine encephalitis virus (EEEV), western equine encephalitis virus (WEEV), Venezuelan equine encephalitis virus (VEEV), $P$. vivax, $P$. malariae, $P$. falciparum, $P$. ovale, $W$. bancrofti, B. malayi and B. timori. Complete genomic sequences of these MBVs, $18 \mathrm{~S}$ rRNA gene sequences of malaria parasites, SspI repeat DNA sequence of $W$. bancrofti, and Hhal repeat region sequences of $B$. malayi and B. timori were downloaded from the GenBank database. The sequences were aligned using AlignX (a component of the Vector NTI Advance 10.3.0) to compare the homology between potential targets belong to the same genus. Gene-specific primers (GSPs) were derived from published literature or designed from conserved regions using Primer Premier 5 (PREMIER Biosoft International, Palo Alto, USA). Reverse primers used in Multiplex RT-PCR or PCR were biotin-labelled at 5' end to form a $\mathrm{CL}$ reaction. The capture probes, which were used to capture the target DNA amplification fragments, were designed by Oligo 7 (Molecular Biology Insights, Inc., Colorado Springs, USA). Continuous homologous sequences of congeners were excluded. A human-original sequence was used as negative control probe. A repeat sequence of $(\mathrm{T})_{12}$ with an amino-labeled $3{ }^{\prime}$-end was connected to the 3 '-end of all the probes to fix the repeat sequence with the aldehyde-chip surface. A repeat sequence of $(\mathrm{T})_{20}$ with a biotin-labeled $5^{\prime}$-end and an aminolabeled 3'-end served as a quality control (QC) probe. All the primers and probes were confirmed by NCBI BLAST and synthesized by Sangon Biotech Co., Ltd. (Shanghai, China).

To evaluate the efficiency of primers, serially diluted viral RNAs or plasmid DNAs were amplified by RT-PCR or PCR and resolved by $2 \%$ agarose gel electrophoresis. Furthermore, the most efficient pairs of primers were biotin labeled in reverse primers, and RT-PCR or PCR was used to evaluate the DNA hybridization efficiency of the capture probes. 


\section{Preparation of in vitro transcript RNA}

To verify the LOD of this strategy and calibrate the concentration of virus-like particles (VLPs), in vitro transcribed RNAs of these MBVs were prepared. First, plasmids were constructed by cloning target gene fragments to PGM-T vector as described in the manufacturer's protocol (Tiangen Biotech Beijing Co., Ltd.). Following digestion with FastDigest Sal I restriction endonuclease (ThermoFisher, Waltham, USA), the plasmids were purified by agarose gel and retrieved. Subsequently, in vitro transcribed RNAs were prepared with TranscriptAid T7 High Yield Transcription Kit (ThermoFisher), digested by deoxyribonuclease (DNase) I, and purified by phenol-chloroform extraction method as described in the manufacturer's protocol (ThermoFisher). Further, in vitro transcribed RNAs were purified and digested by DNase I using RNeasy Mini Kit (QIAGEN, Mississauga, Canada) as described in the manufacturer's protocol. The purified RNAs were mixed with $2 \times$ RNA Loading Dye Solution (ThermoFisher) and resolved by $2 \%$ agarose gel electrophoresis. Finally, in vitro transcribed RNAs were quantified by UV spectrophotometry and subject to serial dilutions at a magnitude of 10 and stored at $-70{ }^{\circ} \mathrm{C}$ until use.

\section{Preparation of virus-like particles}

The maturation protein gene and coat protein gene of MS2 bacteriophage were amplified by MS2-BamH I and MS2-Hind III primers (modified from reference literature) [29], and the sequence was as follows: MS2-BamH I, $5^{\prime}$-AAC GGA TCC CAT GGC TAT CGC TGT AGG TAG CC-3', MS2-Hind III, 5'-CAT AAG CTT CTT CGA CAT GGG TAA TCC TCA TGT T-3'. The bases in italics represent endonuclease sites of $\mathrm{BamH} \mathrm{I}$ and Hind III. The PET-MS2 vector was constructed using the MS2 amplified fragment and PET-28a (+) vector (Novagen, Merck KGaA, Darmstadt, Germany) and ligated using T4 DNA ligase (New England BioLabs ${ }^{\circ}$ Inc., Ipswich, USA) after digestion with restriction enzymes (BamH I and Hind III, ThermoFisher), according to the manufacturer's protocol. The Hind III restriction enzyme site and protection bases were added to the $5^{\prime}$ end of MBVs' GSPs (both forward and reverse), and target genes of these mosquito-borne viruses were amplified and digested by Hind III restriction enzyme. PET-MS2 vectors were also digested by Hind III and dephosphorylated by alkaline phosphatase (New England BioLabs ${ }^{\circ}$ Inc.). Target gene fragments of MBVs and PET-MS2 vectors were ligated using T4 DNA ligase and cloned to BL21 (DE3) competent cells (Tiangen Biotech Beijing Co., Ltd.), according to the manufacturer's instructions. These bacterial plasmids were induced by isopropyl-1thio- $\beta$-galactopyranoside (IPTG) to express VLPs and lysed by sonication. The VLPs were precipitated by
PEG8000 and purified by $15-45 \%(\mathrm{w} / \mathrm{v})$ sucrose density gradient ultracentrifugation at 32,000× rpm for $6 \mathrm{~h} / 4{ }^{\circ} \mathrm{C}$ (Beckman, MAL-80 ROTOR, Brea, USA). The aliquot containing VLPs was dialyzed against PBS at $4{ }^{\circ} \mathrm{C}$ for $24 \mathrm{~h}$ to remove sucrose. The purified VLPs were used as positive RNA references materials.

\section{Multiplex asymmetric RT-PCR and PCR amplification}

The GSPs of MBVs and MBPs were used in two multiplex asymmetric RT-PCR systems and one multiplex asymmetric PCR system, respectively. For optimal amplification efficiency, orthogonal experiment was used to optimize the concentration of the GSPs. Briefly, the concentration ratio of the forward and reverse primers were set to 1:5. The high, medium, and low concentrations of primers were set based on the results of preliminary experiments. The selected primer concentrations were determined by evaluation of the amplification efficiencies of the target genes. Each RT-PCR was performed in a $25 \mu \mathrm{l}$ reaction volume, containing $12.5 \mu \mathrm{l}$ of $2 \times$ One Step Buffer, $1.0 \mu \mathrm{l}$ of PrimeScript One-step Enzyme Mix (DRR055A, Takara Biotechnology (Dalian) Co., Ltd., Dalian, China), $5 \mu \mathrm{l}$ of total RNA template, and specific primer mix. RT-PCR system A amplified the target genes of mosquito-borne flavivirus included DENV, JEV, YFV, SLEV, and WNV. System A contained GSPs of DC10418 $(0.2 \mu \mathrm{mol})$, JEV-3UTR-F2 $(0.28 \mu \mathrm{mol})$, YFV-3UTR-F1 $(0.4 \mu \mathrm{mol})$, WNV-3UTR-F2 $(0.2 \mu \mathrm{mol})$, SLE-3UTR-F1 $(0.1 \mu \mathrm{mol})$, and CDC10564 $(2 \mu \mathrm{mol})$. RT-PCR system B amplified the target genes of mosquito-borne alphavirus included EEEV, WEEV, VEEV, and CHIKV. System B contained GSPs of EEE-E1-F $(0.4 \mu \mathrm{mol})$, EEE-E1-R ( $2 \mu \mathrm{mol})$, CHIKV-F1 $(0.2 \mu \mathrm{mol})$, CHIKV-R2 (1 $\mu \mathrm{mol})$, WEE-F $(0.08 \mu \mathrm{mol})$, WEE-R $(0.4 \mu \mathrm{mol})$, VEEV-cap-F3 $(0.12 \mu \mathrm{mol})$, and VEEV-cap-R3 $(0.6 \mu \mathrm{mol})$. PCR was performed in a $25 \mu \mathrm{l}$ reaction volume containing $2.5 \mu \mathrm{l}$ of 10× EX Taq Buffer, $0.5 \mu \mathrm{l}$ of TaKaRa Ex Taq HS (RR006, Takara Biotechnology (Dalian) Co., Ltd.), $200 \mu \mathrm{mol}$ of each dNTP, $5 \mu$ of total DNA template, and specific primer mix. PCR system amplified target genes of plasmodium and lymphatic filariae. PCR system contained GSPs of MAL-F1 $(0.16 \mu \mathrm{mol})$, MAL-R1 $(0.8 \mu \mathrm{mol}), \mathrm{BM} /$ WBF $(0.08 \mu \mathrm{mol})$, WBR $(0.4 \mu \mathrm{M})$, MGB-HhaI-For $(0.16 \mu \mathrm{mol})$, and MGB-HhaI-Rev $(0.8 \mu \mathrm{mol})$. Amplifications were performed on a Veriti 96-Well Thermal Cycler PCR system (Applied Biosystems, Foster City, USA). RT-PCR was performed under the following conditions: $30 \mathrm{~min}$ at $50{ }^{\circ} \mathrm{C} ; 2 \mathrm{~min}$ at $94{ }^{\circ} \mathrm{C} ; 45$ cycles of $20 \mathrm{~s}$ at $94{ }^{\circ} \mathrm{C}, 20 \mathrm{~s}$ at $55^{\circ} \mathrm{C}$, and $20 \mathrm{~s}$ at $72{ }^{\circ} \mathrm{C}$; and a final extension of $5 \mathrm{~min}$ at $72{ }^{\circ} \mathrm{C}$. PCR was performed under the following conditions: $2 \mathrm{~min}$ at $95{ }^{\circ} \mathrm{C} ; 45$ cycles of $20 \mathrm{~s}$ at $94{ }^{\circ} \mathrm{C}, 20 \mathrm{~s}$ at $55^{\circ} \mathrm{C}$, and $20 \mathrm{~s}$ at $72{ }^{\circ} \mathrm{C}$; and a final extension of $5 \mathrm{~min}$ at $72{ }^{\circ} \mathrm{C}$. 


\section{DNA Hybridization and signal detection}

Captured probes of MBVs and MBPs $(50 \mu \mathrm{mol})$ were used to establish the MBVs and MBPs capture-chip, and the spotting was repeated thrice in the vertical direction on a aldehyde modified glass slide (Baio Technology Shanghai Co., Ltd., Shanghai, China) surface with uniform proportional printing buffer ( $5 \%$ glycerol, $0.1 \%$ sodium dodecyl sulfate (SDS), $6 \times$ saline-sodium citrate buffer (SSC), and 2\% (wt/vol) Ficoll 400) as described in previous studies [30]. The QC probe, used at $12.5 \mu \mathrm{mol}$ final concentration, was spotted and repeated six to seven times in the horizontal direction to calibrate the CL signal values. Each aldehyde slide was divided into 10 blocks $(11 \times 11 \mathrm{~mm})$ by a waterproof film to detect 10 different samples.

After amplification, the two multiplex RT-PCR reaction products, amplified using the same template, were blended. After $5 \mathrm{~min}$ of denaturation at $95{ }^{\circ} \mathrm{C}$, the $5 \mu \mathrm{l}$ of RT-PCR mixtures or PCR products were immediately placed on ice for $5 \mathrm{~min}$ and mixed with $5 \mu \mathrm{l}$ of DNA hybridization buffer $(8 \times$ SSC, $0.6 \%$ SDS, $10 \%$ formylamine, and $10 \times$ Denhardt). DNA hybridization mixtures $(10 \mu \mathrm{l})$ were hybridized on the MBVs or MBPs capture-chip for $1 \mathrm{~h}$ at $45{ }^{\circ} \mathrm{C}$. The capture-chip was washed for $30 \mathrm{~s}$ each with $1 \times \mathrm{SSC}$ and $0.2 \% \mathrm{SDS}$, $0.2 \times$ SSC, and $0.1 \times$ SSC at room temperature. The capture-chip was incubated with $15 \mu \mathrm{l}$ of streptavidinhorseradish peroxidase (Str-HRP, Sigma-Aldrich, St. Louis, USA) for $25 \mathrm{~min}$ at $37{ }^{\circ} \mathrm{C}$. Subsequently, the capture-chip was washed with PBST (phosphate buffer, $0.05 \%$ Tween 20) $10 \mathrm{~s}$ at room temperature. Finally, $10 \mu \mathrm{l}$ of pre-mixed CL HRP substrate luminal solution and $\mathrm{H}_{2} \mathrm{O}_{2}$ (Millipore Corporation, Boston, USA) were added to the capture-chip and detected immediately with a micro-light level imaging system (developed in-house, patent number CN201330010609) (The working principle of this CL imaging DNA hybridization method were showed in Fig. 2c).

\section{Determination of cut-off values of capture probes}

In this assay, low background CL imaging and selfprogramming software were employed as the imaging method and the interpretation software was used to read the grey values of the probes. The grey values recorded by the software were set to be in the range of 0-256. The CL signal values of probes $=$ mean of signal values of probes signal values of background. The CL signal value was calibrated as follows: the calibrated CL signal value of a probe $=$ mean of CL signal value of the probe / mean of $\mathrm{CL}$ signal value of $\mathrm{QC}$ probe (the same detection block) $\times$ 100 . The cut-off values of probes were calculated as follows: cut-off value of a probe $=$ average signal values of to detect other pathogens (except the pathogen detected by this probe) $+3 \times$ standard deviation. Probe signal was considered positive when the value was greater than the cutoff value of the probe.

\section{Specificity and sensitivity}

Due to the lack of MBVs and MBPs samples, the specificity of the strategy was evaluated only by 6 cultivated MBVs strains and an inactivated JEV Vaccine (Table 1). The other MBVs and MBPs were evaluated by artificial VLPs, in vitro transcribed RNAs, or plasmids. Further, a panel of non-MBVs, which include tick-borne encephalitis virus (TBEV), avian influenza A (H5N1), 2009 influenza A (H1N1) (PH1N1), seasonal influenza A (H3N2), seasonal influenza A (H1N1), Hepatitis B virus (HBV), Hepatitis $\mathrm{C}$ virus (HCV), adenovirus AD2, AD3, AD40, rubella virus, mumps virus, and respiratory syncytial viruses (RSV) HK6 and B, were also used to determine the specificity of the strategy.

To evaluate the LOD of this strategy, in vitro transcribed RNAs or plasmids were quantified using UV spectrophotometer, and the copy numbers were calculated, serially diluted to 10 -fold, and were detected. Purified VLPs were also diluted and detected to determine

Table 1 Actual virus samples used in this assay

\begin{tabular}{|c|c|c|c|c|}
\hline No. & Genus & Virus & Type & Source \\
\hline 1 & Flavivirus & Dengue virus 2 & Cultivated strain & $\begin{array}{l}\text { Institute of Microbiology Epidemiology of Academy of Military Medical } \\
\text { Sciences, China }\end{array}$ \\
\hline 2 & & Japanese encephalitis virus & Cultivated strain & National Institute for Viral Disease Control and Prevention, China CDC \\
\hline 3 & & Japanese encephalitis virus & $\begin{array}{l}\text { Inactivated vaccine } \\
\text { strain }\end{array}$ & JEV Vaccine (P3) \\
\hline 4 & & Yellow fever virus & Cultivated strain & $\begin{array}{l}\text { Institute of Microbiology Epidemiology of the Academy of Military Medical } \\
\text { Sciences, China }\end{array}$ \\
\hline 5 & Alphavirus & $\begin{array}{l}\text { Eastern equine encephalitis } \\
\text { virus }\end{array}$ & Cultivated strain & $\begin{array}{l}\text { Institute of Microbiology Epidemiology of the Academy of Military Medical } \\
\text { Sciences, China }\end{array}$ \\
\hline 6 & & $\begin{array}{l}\text { Western equine encephalitis } \\
\text { virus }\end{array}$ & Cultivated strain & $\begin{array}{l}\text { Institute of Microbiology Epidemiology of the Academy of Military Medical } \\
\text { Sciences, China }\end{array}$ \\
\hline 7 & & Chikungunya virus & Cultivated strain & $\begin{array}{l}\text { Institute of Microbiology Epidemiology of the Academy of Military Medical } \\
\text { Sciences, China }\end{array}$ \\
\hline
\end{tabular}


the relative concentrations as compared to in vitro transcribed RNA.

\section{Simulated detection of mosquito samples}

To test simulated mosquito samples, uninfected mosquito pools of 50 Aedes vigilax were spiked with $50 \mu \mathrm{l}$ $10^{6}$ copies/ $\mu$ l part of single or mixed VLPs or plasmids. Then the spiked mosquito pools were homogenized in $500 \mu$ diluent containing $20 \%$ fetal bovine serum in phosphate-buffered saline using T10 basic ULTRATURRAX $^{\circ}$ small dispersing instrument (IKA, Staufen, Germany). The homogenized samples were centrifuged for $5 \mathrm{~min}$ at $12,000 \times \mathrm{rpm}$. Supernatants were removed to clean microfuge tubes. RNA or DNA was extracted from the supernatant using TIANamp Virus RNA Kit (Tiangen Biotech Beijing Co., Ltd.) or TIANamp Genomic DNA Kit (Tiangen Biotech Beijing Co., Ltd.), respectively, as described in the manufacturer's protocol. Subsequently, the extracted RNAs or DNAs were amplified by RT-PCR or PCR systems and detected by the CL imaging DNA hybridization assay.

\section{Results}

\section{Primers and probes design and evaluation}

The amplification efficiency of 81 GSPs, obtained from published literatures, and 82 GSPs designed for this assay were precisely evaluated by serially diluted viral RNAs or plasmid DNAs. Biotin-labeled reverse primers were used for multiplex asymmetric RT-PCR or PCR to evaluate DNA hybridization efficiency of 223 capture probes. Finally, 20 GSPs and 21 probes were chosen to amplify the MBVs and MBPs targeted genes and capture the target DNA amplification fragments (Tables 2 and 3).

\section{Construction of in vitro transcribed RNAs and VLPs}

Nine in vitro transcribed RNAs of MBVs were prepared to determine the LOD of the MBVs detection assay and calibrate the concentration of VLPs. After purification, in vitro transcribed RNAs were verified by agarose gel electrophoresis (Fig. 1a) and quantified by UV spectrophotometry. The copy numbers of these in vitro transcribed RNAs were calculated as: copy numbers $($ copies $/ \mu \mathrm{l})=$ RNA concentration $(\mathrm{ng} / \mu \mathrm{l}) \times$ Avogadro constant $\times 10^{-9} /($ RNA length $\times 340 \mathrm{~g} / \mathrm{mol})$ (Table 4). The in vitro transcribed RNAs were serial diluted to 10 -fold and stored at $-70{ }^{\circ} \mathrm{C}$ until use.

Twelve VLPs containing the amplified target RNAs were also prepared as the positive RNA references of the MBVs detection strategy. RNase A and DNase I digestion on the prepared VLPs showed that the nucleic acid bands between 1,000 and 2,000 bp tolerated both enzymes due to their packaging in the internal section of

Table 2 The sequences of GSPs for multiplex asymmetric RT-PCR and PCR amplification

\begin{tabular}{|c|c|c|c|c|c|}
\hline Pathogen & Primer & Sequence $\left(5^{\prime}\right.$ to $\left.3^{\prime}\right)$ & Location & Reference $^{a}$ & Primer source \\
\hline Dengue virus & DC10418 & TTGAGTAAACYRTGCTGCCTGTAGCTC & $10,392-10,481$ & GQ398257 & [21] \\
\hline JEV & JEV-3UTR-F2 & GGACTGGGTTAACAAATCTG & $10,525-10,544$ & EF107523 & Present study \\
\hline YFV & YEF-3UTR-F1 & AAACTACGGATGGAGAACCG & $10,489-10,508$ & U54798 & Present study \\
\hline WNV & WNV-3UTR-F2 & AAGTTGAGTAGACGGTGCTGC & $10,533-10,553$ & EU249803 & Present study \\
\hline SLEV & SLE-3UTR-F1 & GGATGTCAGGTAAACGGTGCT & $10,451-10,471$ & FJ753286 & Present study \\
\hline Flavivirus & $\mathrm{CDC1} 10564^{\mathrm{b}}$ & GGGTCTCCTCTAACCTCTAGTCCT & $10,569-10,592$ & GQ398257 & [21] \\
\hline \multirow[t]{2}{*}{ EEEV } & EEE-E1-F & ACACTAAATTCACCCTAGTTCGAT & $11,376-11,399$ & AY705241 & [22] \\
\hline & EEE-1-R ${ }^{b}$ & GTGTATAAAATTACTTAGGAGCAGCATTATG & $11,492-11,522$ & AY705241 & \\
\hline \multirow[t]{2}{*}{ CHIKV } & CHIKV-F1 & TGGATGAACATGGAAGTGAA & $7,121-7,140$ & DQ443544 & Present study \\
\hline & 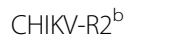 & GCTGTAGTGCGTACCTATTT & $7,496-7,515$ & DQ443544 & Present study \\
\hline \multirow[t]{2}{*}{ WEEV } & WEE-F & AGGGATACCCCCGAAGGTT & $8,220-8,238$ & AF214040 & [23] \\
\hline & WEE- $R^{b}$ & GTGAATAGCACACGGGTGGTT & $8,302-8,322$ & AF214040 & \\
\hline \multirow[t]{2}{*}{ VEEV } & VEEV-cap-F3 & GGGCGGCGCATGAGAGAAGC & $3-22$ & U34999 & Present study \\
\hline & VEEV-cap-R3 ${ }^{\mathrm{b}}$ & GTGACCTGCTTGGCTTCTACCTC & 119-141 & U34999 & Present study \\
\hline \multirow[t]{2}{*}{ Plasmodium } & MAL-F1 & GGGAGTGAAGACGATCAGATACC & $443-465$ & HQ283215 & Present study \\
\hline & $M A L-R 1^{b}$ & CCGTGTTGAGTCAAATTAAGCC & $668-689$ & HQ283215 & Present study \\
\hline \multirow[t]{2}{*}{ W. bancrofti } & BM/WBF & AGCGTGATGGCATCAAAGTAG & $1-21$ & L20344 & [24] \\
\hline & $W_{B R}^{b}$ & AGGTTATACCAAGCAAACAAAAA & 110-132 & L20344 & \\
\hline \multirow[t]{2}{*}{ B. malayi/ B. timori } & MGB-Hhal-For & GCAATATACGACCAGCAC & $254-271$ & M12691 & [25] \\
\hline & MGB-Hhal-Rev ${ }^{b}$ & ACATTAGACAAGGAAATTGGTT & $140-161$ & M12691 & \\
\hline
\end{tabular}

${ }^{\mathrm{a} G e n B a n k}$ accession numbers

${ }^{\mathrm{b}}$ These primers were labeled by biotin to give off $\mathrm{CL}$ signals 
Table 3 Capture probe sequences for DNA hybridization

\begin{tabular}{|c|c|c|c|c|}
\hline Pathogen & Capture probe & Sequence $\left(5^{\prime} \text { to } 3^{\prime}\right)^{a}$ & Location & Reference $^{b}$ \\
\hline DENV & DEN-10731 ${ }^{c}$ & $\begin{array}{l}\text { GGGAGGCCATGCGCCATGGAAGCT } \\
\text { GTACGCATGGTGTAGCAGACT }\end{array}$ & $10,443-10,487$ & GQ398257 \\
\hline DENV-1 & DEN1-X10830 & GCCCAACACCAGGGGAAG & $10,520-10,537$ & FJ687426 \\
\hline DENV-2 & DEN2-X10831 & GCCCAAGGYGAGATGAAGCT & $10,535-10,554$ & GQ398257 \\
\hline DENV-3 & DEN3-X10829 & GGCCCGAGCACTGAGGGAAGC & $10,507-10,527$ & FJ898444 \\
\hline DENV-4 & DEN4-X10714 & CGTAATAATCCCTAGGGAGGCC & $10,380-10,401$ & EF457906 \\
\hline JEV & JEV-10726 & CCCACGGCCCAAGCCTCGTCTAGGAT & $10,703-10,728$ & EF107523 \\
\hline YFV & YFV-10679 & CCTCCGCTACCACCCTCCCAC & $10,667-10,687$ & U54798 \\
\hline WNV & WNV-10768 & AACTTCAAAGCCCAATGTCAGACCACG & $10,650-10,676$ & EU249803 \\
\hline SLEV & SLE-10553 & GTGCAAAGCCCCTCATTCCGACTCG & $10,522-10,546$ & FJ753286 \\
\hline EEEV & EEEV-11424 & GTTCGATGTACTTCCGAGCTATGGTGACGGTGG & $11,393-11,425$ & AY705241 \\
\hline $\mathrm{CHIKV}$ & CHIKV-7427 & ATGGCCACCTITGCAAGCTC & $7,421-7,440$ & DQ443544 \\
\hline WEEV & WEEV-8304 & TCGAATGTCACGTTCCCATGCGACAAAC & $8,277-8,304$ & AF214040 \\
\hline VEEV & VEEV-50 & AAATGGAGAAAGTTCACGTTGACATCGAGG & $42-71$ & U34999 \\
\hline Plasmodium & MAL-1207 & TTAGATTGCTTCCTTCAGTACCTTATGAGA & $547-576$ & HQ283215 \\
\hline P. falciparum1 & PF1-1187 & CTTCGAGGTGACTTTTAGA & $532-551$ & HQ283215 \\
\hline P. falciparum2 & PF2-1182 & AGCATTTCTTAGGGAATGTTGATTTATAT & $573-602$ & HQ283222 \\
\hline P. vivax & PV-1173 & AGAGTTTCTCTTCGGAGTTTA & $525-546$ & HQ283223 \\
\hline P. malariae & PM-1181 & ATTCATATTAATGAGTGTTTCTTTAAGATAGCT & $569-601$ & AF145336 \\
\hline P. ovale & PO-1152 & TGGATGAAAARTTITTAAATAAGAAAATTCCTTTC & $391-425$ & JX977167 \\
\hline W. bancrofti & WB-53 & AGTATGAATGGAATTITAAGCAATTITTTG & $52-82$ & L20344 \\
\hline B. malayi/ B. timori & BM-113 & ATAAGCTTTTITAGTAGTTTTGGCACTTAATT & $184-216$ & M12691 \\
\hline Quality control $^{e}$ & QC & TाTाTाTाTाTाTाTाTाT & & \\
\hline Negative control $^{f}$ & NC & CAGAGATACATTGACC & $1,215-1,230$ & NM_001128925 \\
\hline
\end{tabular}

${ }^{\mathrm{a}} \mathrm{A}$ repeat sequence of $(\mathrm{T})_{12}$ with an amino-labeled 3'-end was conjugated to the 3 '-end of all the capture probes

${ }^{\mathrm{b}}$ GenBank accession numbers

${ }^{\mathrm{C}}$ This capture probe was used to detect all the DENVs

${ }^{\mathrm{d}}$ This capture probe was used to detect all the malarial parasites

${ }^{\mathrm{e}} \mathrm{A}$ repeat sequence of $(\mathrm{T})_{20}$ with an amino-labeled 3 '-end and biotin-labeled 5 '-end were used to calibrate the $\mathrm{CL}$ signal values during DNA hybridization

${ }^{f} A$ human-original sequence was used as negative control

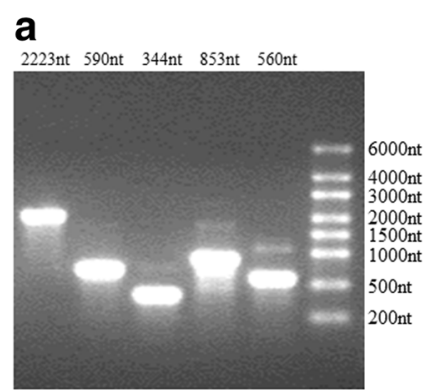

PC CHIKV EEEV YFV JEV RNA Marker b

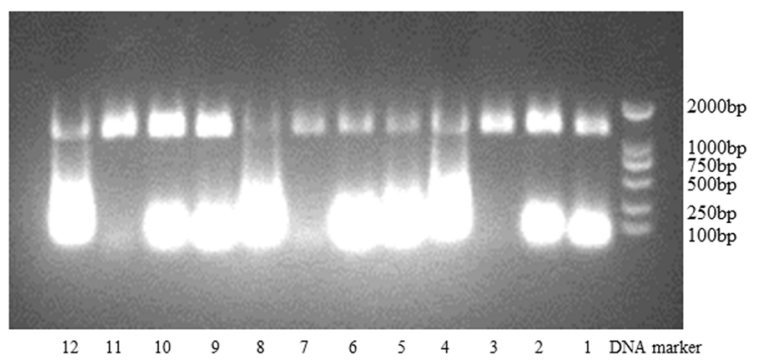

Fig. 1 Determination of in vitro transcribed RNAs and VLPs by agarose gel electrophoresis. a Agarose gel electrophoresis of in vitro transcribed RNAs. The size of the in vitro transcribed RNAs were marked above each lane and compared with a RNA marker (Thermo Fisher). b RNase A and DNase I digestion of VLPS. Lane 1: PET-MS2 (RNase A); Lane 2: PET-MS2 (DNase I); Lane 3: PET-MS2 (RNase A + DNase I); Lane 4: PET-MS2; Lane 5: YFV VLP(RNase A); Lane 6: YFV VLP (DNase I); Lane 7: YFV VLP (RNase A + DNase I); Lane 8: YFV VLP; Lane 9: EEEV VLP (RNase A); Lane 10: EEEV VLP (DNase I); Lane 11: EEEV VLP (RNase A + DNase I); Lane 12: EEEV VLP. The size of VLPs are compared to a DNA marker (TaKaRa). The nucleic acids between 1,000-2,000 bp are resistant to both RNase A and DNase I due to their packaging in the internal section of the VLPS 
Table 4 The copy numbers of in vitro transcribed RNAs quantified by UV spectrophotometer

\begin{tabular}{llllll}
\hline Virus & RNA concentration $(\mathrm{ng} / \mu \mathrm{l})^{\mathrm{a}}$ & Length $(\mathrm{nt})^{\mathrm{b}}$ & Copy number $(\mathrm{copies} / \mu \mathrm{l})^{\mathrm{c}}$ & Location $^{\text {Reference }^{\mathrm{d}}}$ \\
\hline SLEV & 61 & 680 & $2 \times 10^{11}$ & $10,451-10,933$ & FJ753286 \\
WNV & 550 & 654 & $1 \times 10^{12}$ & $10,521-10,977$ & EU249803 \\
VEEV & 275 & 339 & $1 \times 10^{12}$ & $1-142$ & U34999 \\
JEV & 1810 & 560 & $5 \times 10^{12}$ & $10,525-10,887$ & EF107523 \\
YFV & 1930 & 853 & $4 \times 10^{12}$ & $10,109-10,764$ & U54798 \\
EEEV & 1290 & 344 & $6 \times 10^{12}$ & $11,376-11,522$ & AY705241 \\
CHIKV & 1700 & 590 & $5 \times 10^{12}$ & $7,123-7,515$ & DQ443544 \\
WEEV & 510 & 303 & $4 \times 10^{12}$ & $8,217-8,322$ & AF214040 \\
DENV2 & 275 & 395 & $1 \times 10^{12}$ & $10,392-10,589$ & GQ398257 \\
\hline
\end{tabular}

${ }^{a}$ RNA concentrations were quantified by UV spectrophotometer

${ }^{\mathrm{b}}$ The lengths of in vitro transcribed RNAs were calculated on the length of the inserted gene fragments plus the transcript length of digested PGM-T vector ${ }^{c}$ Copy numbers $($ copies $/ \mu \mathrm{l})=$ RNA concentration $(\mathrm{ng} / \mu \mathrm{l}) \times$ Avogadro constant $\times 10^{-9} /(\mathrm{RNA}$ length $\times 340 \mathrm{~g} / \mathrm{mol})$

${ }^{\mathrm{d}}$ GenBank accession numbers

VLPs (Fig. 1b). To determine whether the target RNAs had been packaged in the internal regions, RNase A and DNase I digested VLPs were serially diluted (10-fold), total RNA was extracted, amplified by both multiplex RT-PCR and PCR (both based on the GSPs of RT-PCR system A or B), and detected by MBVs hybridization capture-chip. The results showed that DNase I did not completely digested the DNA templates from high concentration VLPs; the extractions from low-concentration VLPs could only be detected by RT-PCR but not PCR

\begin{tabular}{|c|c|c|c|c|c|c|}
\hline$p$ & p & $p$ & $p$ & $p$ & $p$ & $p$ \\
\hline DEN-10731 & DEN1-X10830 & DEN2-X10831 & DEN3.X10829 & DENA $\times 10714$ & JEV-10726 & YEV-10679 \\
\hline DEN-10731 & DEN1-X10830 & DEN2:X10831 & DEN3.X10829 & DEN4 $\times 10714$ & JEV-10726 & YEV-10679 \\
\hline DEN-10731 & DEN1-X10830 & DEN2-X10831 & DEN3.X10829 & DEN4.X10714 & JEV-10726 & YEV-10679 \\
\hline WNN-10768 & SLE-10553 & EEEV-11424 & WEEV-8304 & VEEV-50 & CHIKV-7427 & NC \\
\hline WNV-10768 & SLE-10553 & EEEV.11424 & WEEV-8304 & VEEY.50 & CHIKV-7427 & NC \\
\hline WNV-10768 & SLE-10553 & EEEV-11424 & WEEV-8304 & VEEY.50 & CHIKV-7427 & NC \\
\hline
\end{tabular}

b

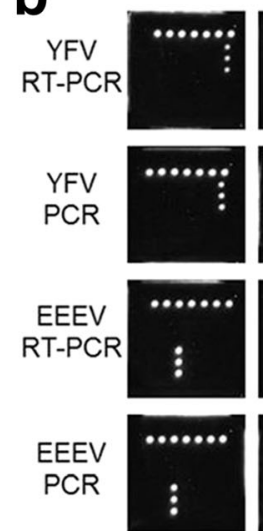

Dilution

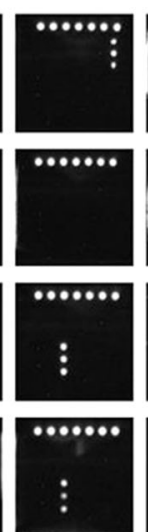

$10^{-5}$

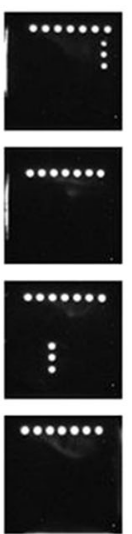

$10^{-6}$

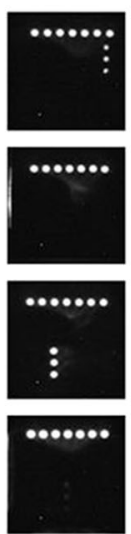

$10^{-7}$
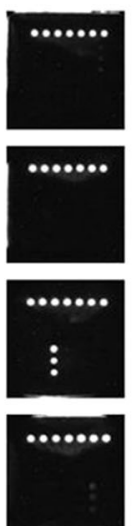

$10^{-8}$
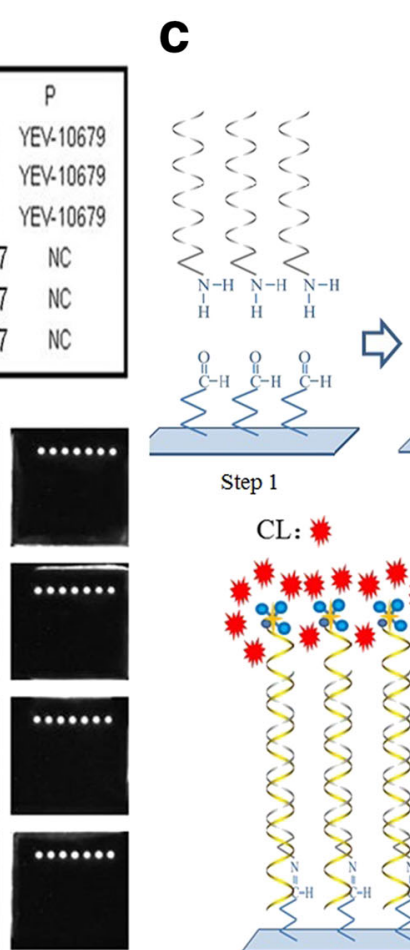

NC

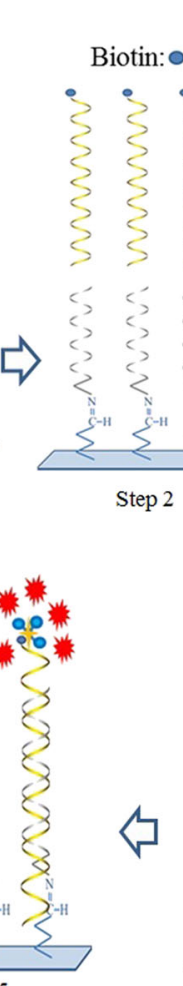

Step 5 treptavidin: + Horseradish peroxidase:

Fig. 2 Amplification of serially diluted VLPs by both RT-PCR and PCR. a The layout of the MBVs hybridization capture-chip. The probe P is the QC probe. The probe NC is the negative control probe. $\mathbf{b}$ Amplifications of serially diluted VLPS by both RT-PCR and PCR (both based on the GSPs of RTPCR system A or B). The results showed that although DNase I did not completely digest DNA templates in high concentration VLPS, the extraction of low-concentration VLPs were only detected by RT-PCR and not by PCR, demonstrating that DNA templates had been diluted to sufficiently. $\mathbf{c}$ Principle of the CL imaging DNA hybridization method. Step 1 shows that capture probes are fixed to the aldehyde-chip surface. Step 2 shows that the denatured RT-PCR products are hybridized on the capture-chip. Steps 3-5 show the principle of CL detection. Biotin is incorporated into reverse strand in RT-PCR amplification. When HRP modified streptavidin is bound, CL signal becomes illuminant by the catalyzed substrates 
(Fig. 2b), thereby demonstrating that the DNA templates had been diluted to sufficiently low concentrations that cannot be detected.

\section{Determination of cut-off value of capture probes}

In this assay, the cut-off values of a probe were calculated as follows, cut-off = average signal value to detect other pathogens (except the particular pathogen detected by this probe) $+3 \times$ standard deviation. The cut-off values of all the probes are shown in Table 5.

\section{Evaluation of specificity}

The specificity of the strategy was evaluated using cultivated MBVs strains, inactivated JEV vaccine strain, artificial VLPs, in vitro transcribed RNAs, and plasmids. The results showed that this assay genotyped MBVs and MBPs with no cross-reactions between the samples and reference materials (Fig. 3a, b). The CL signal value (i.e. that had not been calibrated) of each probe was shown in 3-D bar chart (Fig. 3c, d). Furthermore, a panel of non-MBVs did not yield any positive

Table 5 Cut-off values of the capture probes

\begin{tabular}{|c|c|c|c|}
\hline Probe & $\begin{array}{l}\text { Average of negative } \\
\text { sample (grey value) }\end{array}$ & $\begin{array}{l}\text { SD } \\
\text { (grey value) }\end{array}$ & $\begin{array}{l}\text { Cut-off value } \\
\text { (grey value) }\end{array}$ \\
\hline DEN-10731 & 0.50 & 0.67 & 2.51 \\
\hline DEN1-X10830 & 0.18 & 0.33 & 1.17 \\
\hline DEN2-X10831 & 0.85 & 1.55 & 5.50 \\
\hline DEN3-X10829 & 0.38 & 0.29 & 1.25 \\
\hline DEN4-X10714 & 0.15 & 0.22 & 0.81 \\
\hline JEV-10726 & 0.42 & 0.30 & 1.32 \\
\hline YFV-10679 & 1.90 & 3.80 & 13.30 \\
\hline WNV-10768 & 0.30 & 0.39 & 1.47 \\
\hline SLE-10553 & 0.19 & 0.14 & 0.61 \\
\hline EEEV-11424 & 0.29 & 0.43 & 1.58 \\
\hline WEEV-8304 & 0.64 & 0.85 & 3.19 \\
\hline VEEV-50 & 1.02 & 2.03 & 7.11 \\
\hline CHIKV-7427 & 0.23 & 0.37 & 1.34 \\
\hline MAL-1207 & 0.50 & 0.20 & 1.10 \\
\hline PF1-1187 & 0.10 & 0.14 & 0.52 \\
\hline PF2-1182 & 0.32 & 0.20 & 0.92 \\
\hline PV-1173 & 0.25 & 0.11 & 0.58 \\
\hline PM-1181 & 0.20 & 0.14 & 0.62 \\
\hline PO-1152 & 0.20 & 0.14 & 0.62 \\
\hline BM-113 & 0.43 & 0.42 & 1.69 \\
\hline WB-53 & 0.20 & 0.14 & 0.62 \\
\hline
\end{tabular}

Abbreviation: $S D$ standard deviation

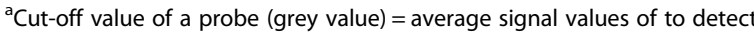
other pathogens (except the particular pathogen detected by this probe) + $3 \times$ standard deviation. A probe signal was considered positive when the value was greater than the cut-off value of a given probe results with this strategy, suggesting the method is indeed highly specific (Additional file 1: Figure S1).

\section{Determination of LOD}

To determine the absolute LOD of this strategy, serially diluted in vitro transcribed RNAs or plasmids, which had been quantified by the copy numbers, served as the reference materials. The absolute LODs for the MBVs were $10^{2}$ copies/ $\mu$ l except for WNV $\left(10^{3}\right.$ copies $\left./ \mu \mathrm{l}\right)$, (Fig. 4a). The calibrated CL signal values of the in vitro transcribed RNAs are described in a line chart (Fig. 4c). Further, the absolute LODs for P. falciparum, P. ovale, and $P$. malariae are $10^{1}$ copies/ $\mu \mathrm{l}$; for the others, the values are $10^{2}$ copies/ $\mu$ l (Fig. 5a). The calibrated CL values of these plasmid DNAs are also described in a line chart (Fig. 5b)

A strain of JEV virus derived from National Institute for Viral Disease Control and Prevention was also employed as a reference material to determinate the LOD of this strategy. The results showed that the LOD for JEV was between $10^{1.8} \mathrm{PFU} / \mathrm{ml}$ and $10^{0.8} \mathrm{PFU} / \mathrm{ml}$ (Fig. 4b). A linear regression model based the calibrated CL signal values against the JEV concentrations shows satisfactory fitness, with the coefficient of determination being 0.9678 (Fig. 4d).

\section{Simulated detection of mosquito samples}

To test simulated mosquito samples, uninfected mosquito pools of 50 Aedes vigilax were spiked with $10^{6}$ copies/ $\mu \mathrm{l}$ of six single VLPs and plasmids or mixed VLPs and plasmids, followed by the CL imaging DNA hybridization assay. The results showed that this assay could detect all mimic samples of single MBVs, single MBPs, mixed mosquito-borne flavivirus, mixed mosquito-borne alphavirus, and mixed MBPs (Fig. 6). Therefore, the proposed assay can potentially test targeted MBVs and MBPs in actual mosquito samples.

\section{Discussion}

As a competent vector, arthropods can support the sufficiently large inoculum of the virus and transmit the MBVs from an infected donor to a recipient host during blood-feeding. MBVs are also transmitted between male and female mosquitoes or from a female to the offspring. Furthermore, malaria and lymphatic filariasis, transmitted by hematophagous arthropods, are still the most prevalent mosquito-borne parasitic diseases in humans $[13,14]$. A continuous effort to monitor the MBVs is critical to prevent sudden outbreaks [20]. Many genebased diagnostic methods have been used to detect MBVs and MBPs. However, to the best of our knowledge, a portable, ultra-sensitive CL DNA hybridization method to simultaneously detect common MBVs and 


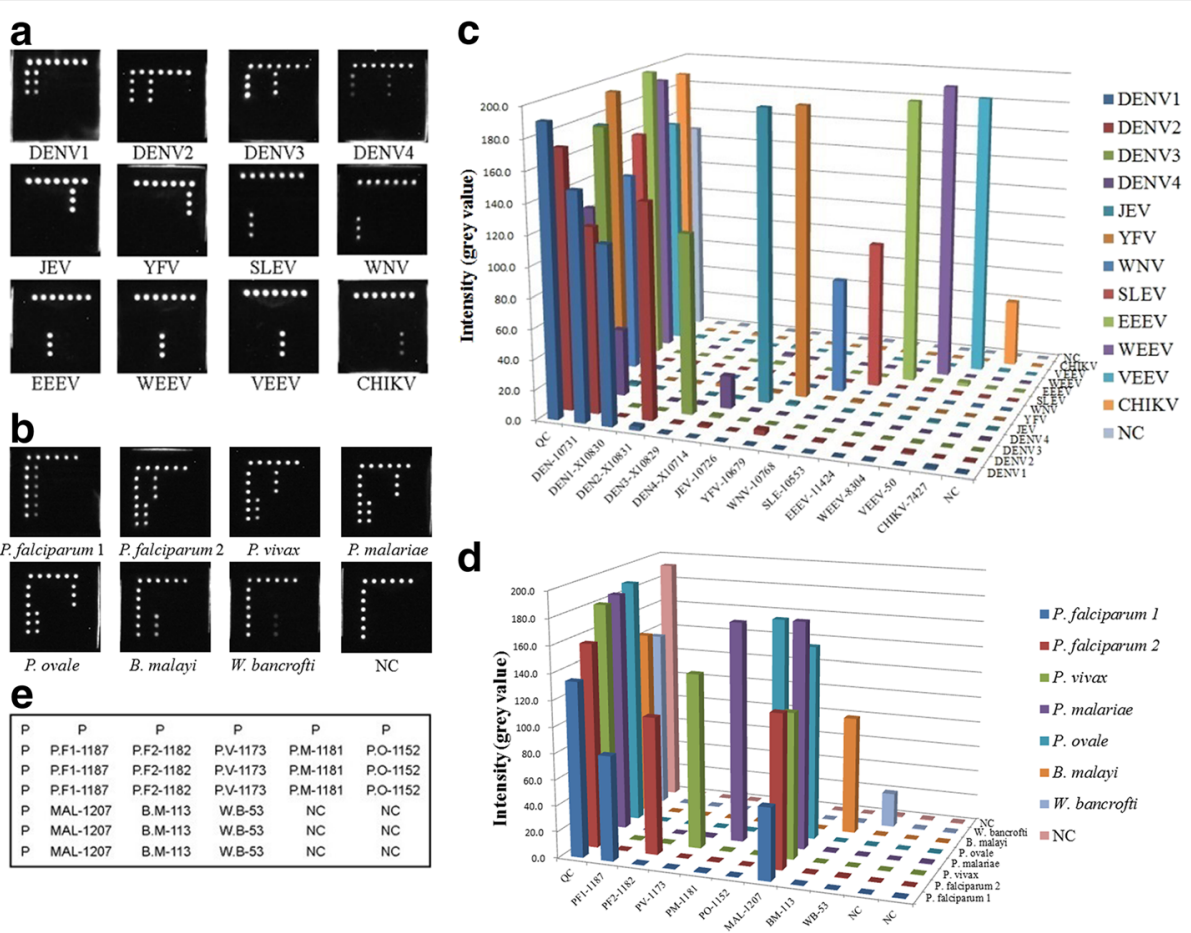

Fig. 3 Specificity of the CL imaging DNA hybridization method. $\mathbf{a}$ and $\mathbf{b}$ Specificity of MBV and MBP detection, respectively. Cross-reaction is not present in samples and reference materials. c CL signal value (not calibrated) of each probe for MBVs is shown in the 3-D bar chart. $\mathbf{d}$ CL signal value (not calibrated) of each probe for the MBPs is shown in the 3-D bar chart. e Layout of the MBPs hybridization capture-chip. The probe P is the QC probe. The probe NC is the negative control probe

MBPs has not been reported. Because of the lack of antiviral drugs $[10,31,32]$, the use of vaccines has emerged as an efficient strategy to control the spread of MBV diseases [33]. At present, human vaccines for flavivirus and alphavirus are available; however, these only include prevention of YFV, JEV, and TBEV [34-38], while the human vaccines for the prevention of DENV, WNV, CHIKV, SLEV, WEEV, EEEV and VEEV are still not approved for clinical trials [33, 39]. As a result, it has become imperative to develop reliable methods for rapid diagnosis, timely prevention, and efficient control of the mosquito-borne diseases. Rapid diagnosis is not only the indispensable method for controlling the infectious diseases, but also the prerequisite for timely prevention and efficient treatment. Infectious diseases are only able to be effectively prevented and treated after rapid and accurate diagnosis. Therefore, the spread of these infectious diseases, for which the drugs are not yet established, can be effectively reduced by controlling their vectors and accurate monitoring. In this assay, we developed a portable, ultra-sensitive CL imaging DNA hybridization method to detect various MBVs and MBPs simultaneously. This method is based on horseradish peroxidase catalyzing luminol- $\mathrm{H}_{2} \mathrm{O}_{2}$, and its performance proved to be highly sensitive and specific for the 12 MBVs and 7 MBPs that were included in the current study. The traditional epidemic of these pathogens involves many tropical and sub-tropical areas of Asia, Africa, the Americans, Europe and the Pacific archipelago [40-43]. Currently, there may not be a region in the world where all 19 pathogens are present. So the inclusion of the 19 pathogens in the current study appears exceeding the needs of clinical detections. However, we believe our study delivers a proof-of-concept study where such a diagnostic assay may test for more pathogens and save significant labour and time when a patient presents with symptoms of unknown etiology. Furthermore, given the fact that mosquito-borne diseases may occur in non-endemic areas due to the uncertainties of global human migration, for example, the first imported cases of Zika virus in China and Europe [44, 45], a rapid and ready-to-be-applied method will have significant clinical implications. For point of care testing, a selfpowered portable CL CCD imager was developed. A self-programming software was employed to interpret the software readings and to study the grey values of the probes. However, other commercial CL imagers based on CCD imaging technology can also be used for CL imaging. Despite needing a CL imager, this CL imaging method has lower cost and faster detection speed than our previous visual method which was based on quantum dot-catalyzed silver deposition [30]. 


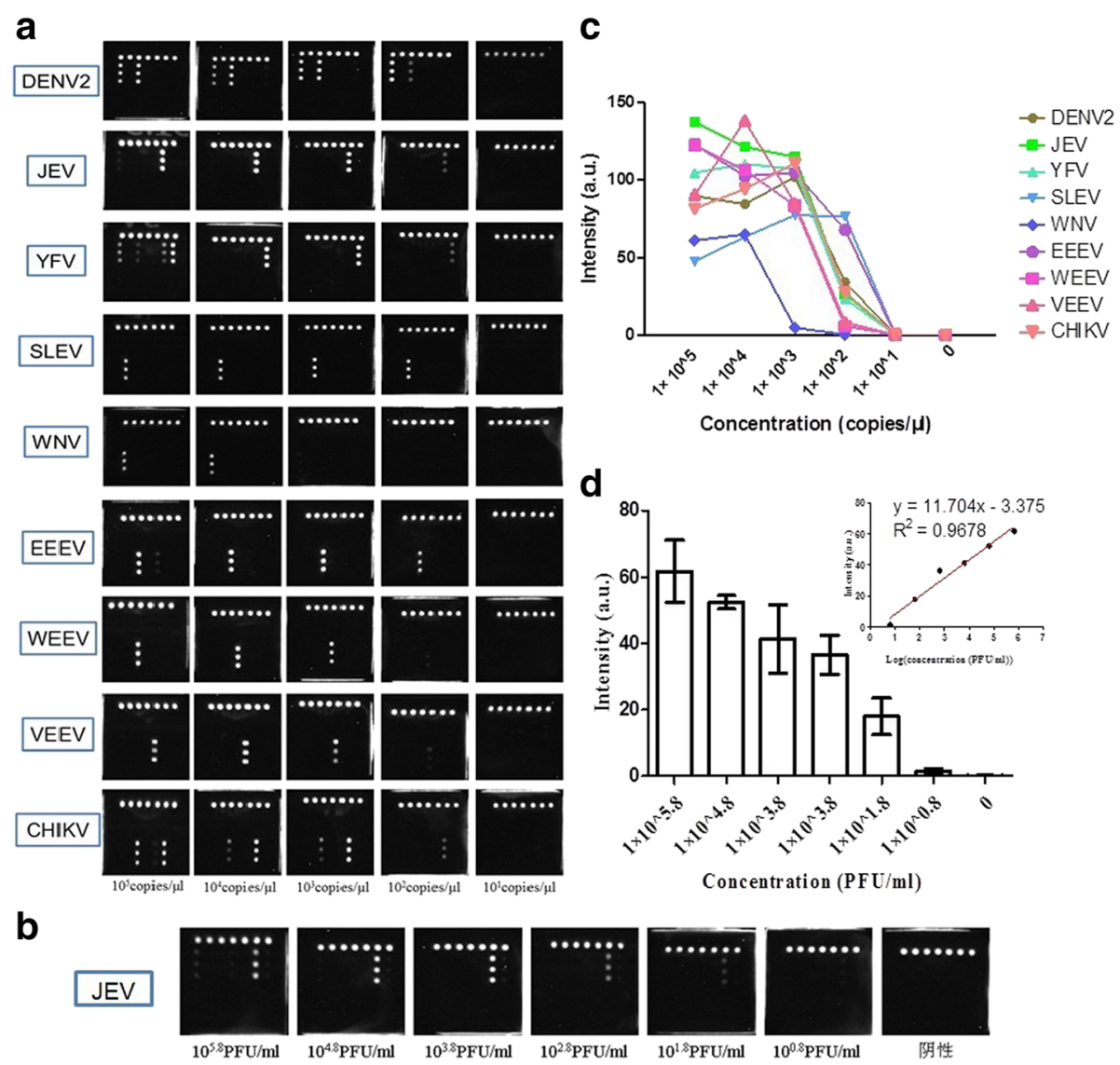

Fig. 4 Determination of LOD for MBVs detection. a Detection of serially diluted in vitro transcribed RNAs of MBVs. The results show that the absolute LOD of this strategy to detect these MBVs is $10^{2}$ copies/ $\mu$ l except for WNV $\left(10^{3}\right.$ copies/ $\left.\mu l\right)$. $\mathbf{b}$ Detection of a strain of JEV that had been accurately quantified by the National Institute for Viral Disease Control and Prevention. The LOD to detect JEV is between $10^{1.8}-10^{0.8} \mathrm{PFU} / \mathrm{ml}$. c Calibrated CL signals of diluted in vitro transcribed RNAs of MBVs. $\mathbf{d}$ Calibrated CL signal values are plotted as a linear function of JEV concentrations (inset). It fits well in a linear regression model. The coefficient of determination is 0.9678 . Data shown are representative of two independent experiments

In the current study, due to the lack of pathogen nucleic acid samples, we validated the specificity of our method using the positive and negative references. The absolute LOD, which was evaluated using serially diluted in vitro transcribed RNAs and plasmids, was in the range of $10^{1}-10^{3}$ copies/ $\mu$ l. A strain of JEV was also employed as a reference material to determine the absolute LOD. Results from the two repeat tests showed that the absolute LOD for JEV was in the range of $10^{0.8}$ $10^{1.8} \mathrm{PFU} / \mathrm{ml}$, and fits well in a linear regression model, demonstrating the feasibility of quantitative measurement of viral load. The assay is rapid, portable, ultrasensitive, and high-throughput. The entire detection time, spanning from sample extraction to result reading, is between 6-8 $\mathrm{h}$.

Since most of the targets in this assay are potent pathogens, we did not collect actual samples or all the nucleic acids. Instead, 14 artificial target pathogen gene sequences were constructed by overlap extension method and cloned into plasmid vectors as well as those target pathogens having nucleic acids samples (datas not shown). Subsequently, RNAs of these target viruses were in vitro transcribed from these plasmids. We observed that DNA templates from the plasmids were not completely removed by DNase I digestion. Consequently, RNeasy Mini Kit (QIAGEN), a purification method based on spin column, was also employed to purify and digest these in vitro transcribed RNAs on column. Although residual DNA templates were not completely removed from in vitro transcribed RNAs, they were negligible after the concentrations of in vitro transcribed RNAs were diluted to lower than $10^{8}$ copies/ $\mu$ l due to the twice DNase I digestion (dates not shown). Similar issue was observed during the preparation of VLPs of these target MBVs. However, to maintain the integrity, the VLPs were not digested by DNase I on the spin column. Consequently, the VLPs, as RNA positive reference materials, were also diluted to a sufficiently low concentration to overcome the influence of residual DNA templates as the concentration of 


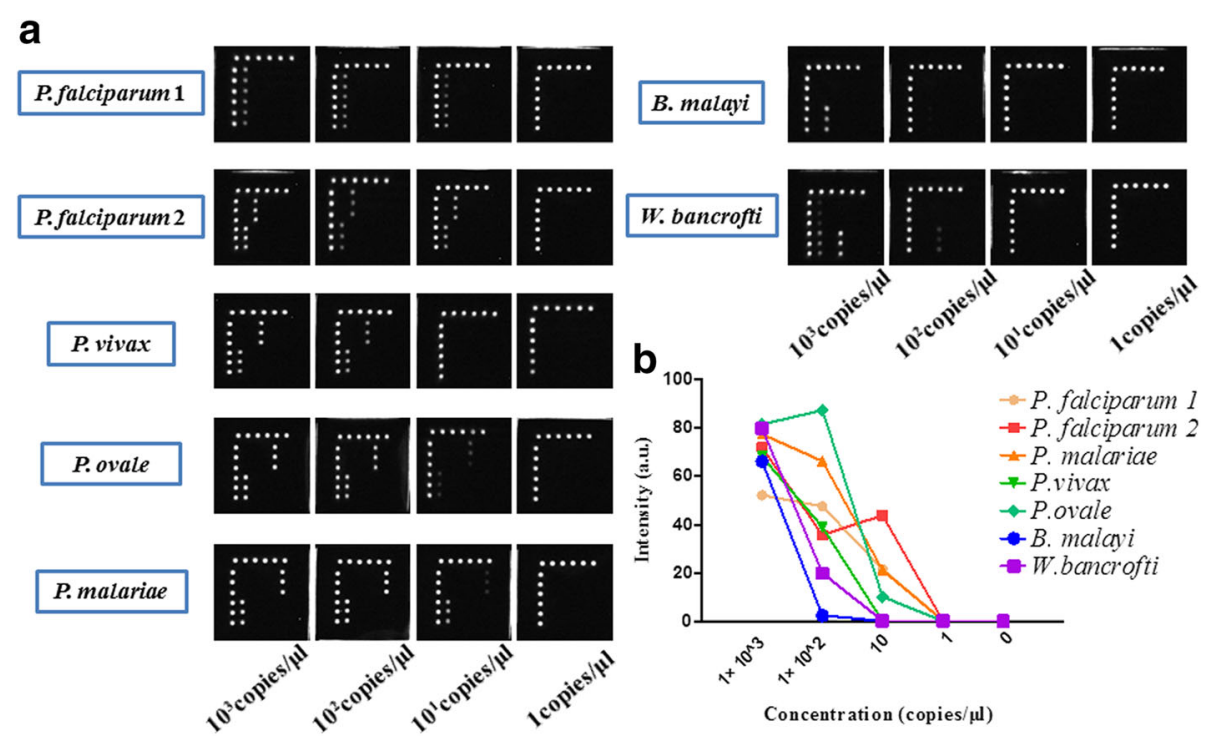

Fig. 5 Determination of LOD for MBPs detection. a Absolute LODs for P. falciparum, P. ovale, and P. malariae is $10^{1}$ copies $/ \mu$ land $10^{2}$ copies $/ \mu l$ for the remaining species. $\mathbf{b}$ Calibrated $C L$ values of plasmid DNAs are shown in a line chart

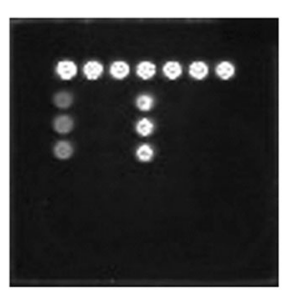

DENV3

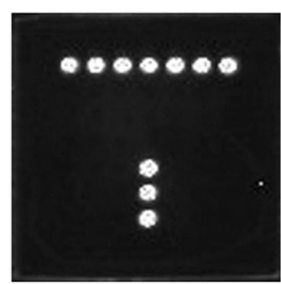

WEEV

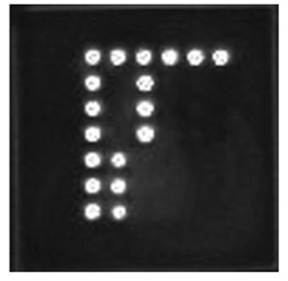

P. falciparum 2

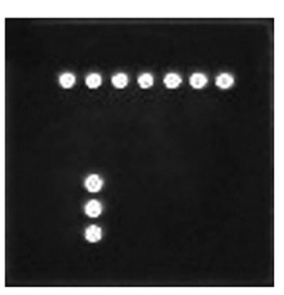

SLEV

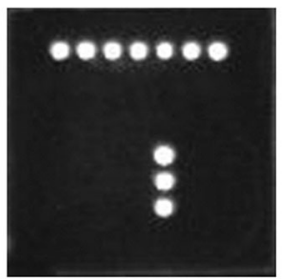

VEEV

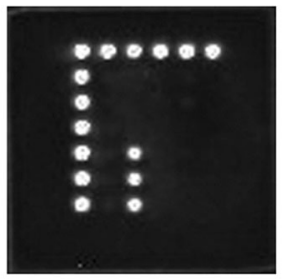

B. malayi

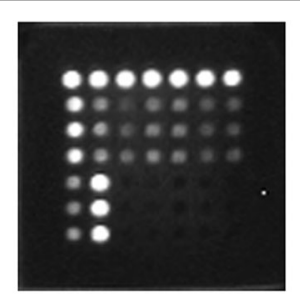

Mixed Flavivirus

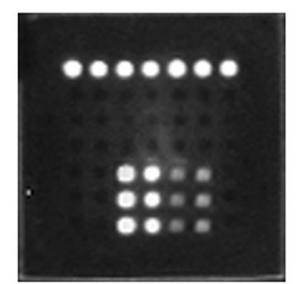

Mixed Alphavirus

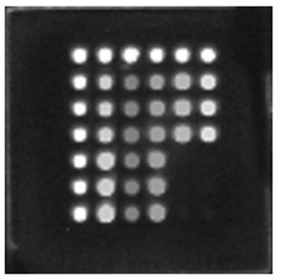

Mixed MBPs

Fig. 6 Simulated detection of mosquito samples. Uninfected mosquito pools of 50 Aedes vigilax were spiked with $10^{6} \mathrm{copies} / \mu \mathrm{l}$ of single VLPS (DENV3, SLEV, WEEV, or VEEV), single plasmids (P. falciparum or B. malayi), mixed VLPS of mosquito-borne flavivirus (including DENV1-4, JEV, YFV, SLEV, and WNV), mixed mosquito-borne alphavirus (including EEEV, WEEV, VEEV and CHIKV), or mixed plasmids of MBPs (including $P$. falciparum, $P$. vivax, P. malariae, P. ovale, W. bancrofti and B. malayi). This assay could detect single MBVs and MBPs in the mimic samples, as well as mixed mosquito-borne flavivirus, mixed mosquito-borne alphavirus, and mixed MBPs 
residual DNAs were only $10^{3}-10^{4}$-fold lower than that of the RNAs within VLPs.

In the present assay, a uniform reverse primer located in the 3'UTR region of flavivirus genome was employed in the multiplex asymmetric RT-PCR. Consequently, relatively shorter probes were used for flavivirus to eliminate cross signals among flavivirus species due to the homology of the genomic sequences of the 3'UTRs. On the other hand, our method did not distinguish between $B$. malayi and B. timori because the target sequences (i.e. HhaIrepeat sequence) are almost identical. Furthermore, methods based on DNA hybridization inevitably appeared cross signal with off target capture probes, especially for the samples containing extremely high concentrations of the target pathogens. Consequently, the cut-off values of capture probes showed in Table 5 need to be adjusted and refined to test the different concentrations of the target pathogen for future application. At present, there are many commercial extraction kits available to extract genomic RNA and DNA from diverse origins, so the proposed strategy can potentially detect these MBVs and MBPs regardless of the origins of these pathogens. In the proof-of-concept experiment, we spiked uninfected mosquito pools with VLPs or plasmids, demonstrated that our CL imaging DNA hybridization method could detect target MBVs and MBPs in mosquito samples. Therefore, further research should be focused on extending the method into the clinical settings for the epidemiological investigations of MBVs and MBPs.

\section{Conclusions}

Ultra-sensitive CL imaging DNA hybridization was developed and could simultaneously detect MBVs and MBPs. This method is based on horseradish peroxidase catalyzing luminol- $\mathrm{H}_{2} \mathrm{O}_{2}$, and its performance proved to be rapid, portable, high-throughput, highly sensitive and specific. The entire operation time, spanning from sample extraction to result reading, is between $6-8 \mathrm{~h}$. The method described here has the potential to provide considerable labor savings due to its ability to screen for 19 mosquito-borne pathogens simultaneously.

\section{Additional file}

Additional file 1: Figure S1. Determination of the specificity of the MBVs detection by detection of non-MBVs. A panel of non-MBVs were detected by this MBVs detection strategy to determine the specificity. There were no positive results (i.e. detection of these non-MBVs). YFV and EEEV were used as positive references. (XML 7 kb)

\section{Abbreviations}

CHIKV: Chikungunya virus; CL: Chemiluminescence; DENV: Dengue virus; DNase: Deoxyribonucleas; EEEV: Eastern equine encephalitis virus; GSPs: Gene-specific primers; HBV: Hepatitis B virus; HCV: Hepatitis C virus; JEV: Japanese encephalitis virus; LOD: Limit of detection; MBPs: Mosquitoborne parasites; MBVs: Mosquito-borne viruses; QC: Quality control;
RSV: Respiratory syncytial viruses; SDS: Sodium dodecyl sulfate; SLEV: St. Louis encephalitis virus; SSC: Saline-sodium citrate buffer; TBEV: Tick-borne encephalitis virus; VEEV: Venezuelan equine encephalitis virus; VLPS: Virus-like particles; WEEV: Western equine encephalitis virus; WHO: World Health Organization; WNV: West Nile virus; YFV: Yellow fever virus

\section{Acknowledgements}

We thank the Institute of Microbiology Epidemiology of Academy of Military Medical Sciences, National Institute for Viral Disease Control and Prevention and the $302^{\text {nd }}$ Hospital of the Chinese People's Liberation Army for providing cultivated samples of MBVs, non-MBVs and Uninfected mosquito pools.

\section{Funding}

This work was supported by the National Science and Technology Major Project (No. 2013ZX10004-101 and No. 2015ZX09J15105-001-001), the Science and Technology Major of Guangdong Province (No. 2012A080203005) and the Science and Technology Program of Tianjin (No. 15PTGCSY00150). The funders had no role in study design, data collection and analysis, decision to publish, or preparation of the manuscript.

\section{Availability of data and materials}

The datasets supporting the conclusions of this article are included within Additional file 1

\section{Authors' contributions}

YZ and QL wrote the main manuscript text; BZ participated in the experiment; XW participated in the experimental results discussed; SC and SW guided and developed the experimental program. All authors read and approved the final manuscript.

\section{Competing interests}

The authors declare that they have no competing interests.

\section{Consent for publication}

Not applicable.

Ethics approval and consent to participate Not applicable.

\section{Author details}

'Beijing Institute of Radiation Medicine, Beijing 100850, People's Republic of China. ${ }^{2}$ Beijing Key Laboratory of New Molecular Diagnosis Technologies for Infectious Diseases, Beijing 100850, People's Republic of China. ${ }^{3}$ Postdoctoral Research Workstation, 210th Hospital of the Chinese People's Liberation Army, Dalian 116021, People's Republic of China.

Received: 10 May 2016 Accepted: 7 January 2017

Published online: 25 January 2017

\section{References}

1. Hubalek Z. Mosquito-borne viruses in Europe. Parasitol Res. 2008;103 Suppl 1:S29-43.

2. Weissenbock H, Hubalek Z, Bakonyi T, Nowotny N. Zoonotic mosquitoborne flaviviruses: worldwide presence of agents with proven pathogenicity and potential candidates of future emerging diseases. Vet Microbiol. 2010; 140(3-4):271-80.

3. Ho PS, Ng MM, Chu JJ. Establishment of one-step SYBR green-based real time-PCR assay for rapid detection and quantification of chikungunya virus infection. Virol J. 2010;7:13.

4. Palani G, Padmanabhan PP, Ramesh K, Asadullah KS, Sambasivam M, Arunagiri K, Krishnasamy K. Development and evaluation of flaviimmunoglobulin M capture enzyme-linked immunosorbent assay. Indian J Pathol Micr. 2013:56(3):269-71.

5. Gould EA, Higgs S. Impact of climate change and other factors on emerging arbovirus diseases. T Roy Soc Trop Med H. 2009;103(2):109-21.

6. Lanciotti RS, Roehrig JT, Deubel V, Smith J, Parker M, Steele K, et al. Origin of the West Nile virus responsible for an outbreak of encephalitis in the northeastern United States. Science. 1999;286(5448):2333-7.

7. Murray KO, Ruktanonchai D, Hesalroad D, Fonken E, Nolan MS. West Nile virus, Texas, USA, 2012. Emerg Infect Dis. 2013;19(11):1836-8. 
8. Wu D, Wu J, Zhang Q, Zhong H, Ke C, Deng X, et al. Chikungunya outbreak in Guangdong Province, China, 2010. Emerg Infect Dis. 2012;18(3):493-5.

9. Arankalle VA, Shrivastava S, Cherian S, Gunjikar RS, Walimbe AM, Jadhav SM, et al. Genetic divergence of Chikungunya viruses in India (1963-2006) with special reference to the 2005-2006 explosive epidemic. J Gen Virol. 2007;88(Pt 7):1967-76.

10. World Health Organization and the Special Program for Research and Training in Tropical Diseases (TDR). Dengue Guidelines for Diagnosis, Treatment, Prevention and Control: New Edition. 2009.

11. Bhatt S, Gething PW, Brady OJ, Messina JP, Farlow AW, Moyes CL, et al. The global distribution and burden of dengue. Nature. 2013;496(7446):504-7.

12. Gubler DJ. The economic burden of dengue. Am J Trop Med Hyg. 2012; 86(5):743-4.

13. Birkholtz LM, Bornman R, Focke W, Mutero C, de Jager C. Sustainable malaria control: transdisciplinary approaches for translational applications. Malaria J. 2012:11:431.

14. Manguin S, Bangs MJ, Pothikasikorn J, Chareonviriyaphap T. Review on global co-transmission of human Plasmodium species and Wuchereria bancrofti by Anopheles mosquitoes. Infect Genet Evol. 2010;10(2):159-77.

15. Kyelem D, Biswas G, Bockarie MJ, Bradley MH, El-Setouhy M, Fischer PU, et al. Determinants of success in national programs to eliminate lymphatic filariasis: a perspective identifying essential elements and research needs. Am J Trop Med Hyg. 2008;79(4):480-4.

16. Molyneux DH, Zagaria N. Lymphatic filariasis elimination: progress in global programme development. Ann Trop Med Parasit. 2002;96 Suppl 2:515-40.

17. Buck AA, Anderson RI, MacRae AA. Epidemiology of poly-parasitism. I. Occurrence, frequency and distribution of multiple infections in rural communities in Chad, Peru, Afghanistan, and Zaire. Tropenmed Parasitol. 1978;29(1):61-70

18. World Malaria Report 2008. http://www.who.int/malaria/publications/atoz/ 9789241563697/en/. Accessed 1 Jan 2008.

19. Lymphatic filariasis. http://www.who.int/mediacentre/factsheets/fs102/en/. Accessed 1 Feb 2016.

20. Gould EA, Higgs S, Buckley A, Gritsun TS. Potential arbovirus emergence and implications for the United Kingdom. Emerg Infect Dis. 2006;12(4):549-55.

21. Chien LJ, Liao TL, Shu PY, Huang JH, Gubler DJ, Chang GJ. Development of real-time reverse transcriptase PCR assays to detect and serotype denque viruses. J Clin Microbiol. 2006;44(4):1295-304.

22. Hull R, Nattanmai S, Kramer LD, Bernard KA, Tavakoli NP. A duplex real-time reverse transcriptase polymerase chain reaction assay for the detection of St. Louis encephalitis and eastern equine encephalitis viruses. Diagn Micr Infec Dis. 2008;62(3):272-9.

23. Kang X, Li Y, Liu H, Lin F, Cai X, Sun T, et al. A duplex real-time reverse transcriptase polymerase chain reaction assay for detecting western equine and eastern equine encephalitis viruses. Virol J. 2010;7:284.

24. Mishra K, Raj DK, Hazra RK, Dash AP, Supakar PC. The development and evaluation of a single step multiplex PCR method for simultaneous detection of Brugia malayi and Wuchereria bancrofti. Mol Cell Probe. 2007; 21(5-6):355-62.

25. Rao RU, Weil GJ, Fischer K, Supali T, Fischer P. Detection of Brugia parasite DNA in human blood by real-time PCR. J Clin Microbiol. 2006;44(11):3887-93.

26. Lanciotti RS, Kerst AJ. Nucleic acid sequence-based amplification assays for rapid detection of West Nile and St. Louis encephalitis viruses. J Clin Microbiol. 2001;39(12):4506-13.

27. Xiao-Ping K, Yong-Qiang L, Qing-Ge S, Hong L, Qing-Yu Z, Yin-Hui Y. Development of a consensus microarray method for identification of some highly pathogenic viruses. J Med Virol. 2009;81(11):1945-50.

28. Hu Z, Nordstrom H, Nowotny N, Falk Kl, Sandstrom G. Anchored pan dengue RT-PCR and fast sanger sequencing for detection of dengue RNA in human serum. J Med Virol. 2010;82(10):1701-10.

29. Huang J, Yang CM, Wang LN, Meng S, Deng W, Li JM. A novel real-time multiplex reverse transcriptase-polymerase chain reaction for the detection of HIV-1 RNA by using dual-specific armored RNA as internal control. Intervirology. 2008:51(1):42-9.

30. Zhang Y, Liu Q, Wang D, Chen S, Wang S. Simultaneous detection of oseltamivir- and amantadine-resistant influenza by oligonucleotide microarray visualization. PLoS One. 2013;8(2):e57154.

31. Plummer EM, Shresta S. Mouse models for dengue vaccines and antivirals. J Immunol Methods. 2014;410:34-8.

32. Reichert E, Clase A, Bacetty A, Larsen J. Alphavirus antiviral drug development: scientific gap analysis and prospective research areas. Biosecur Bioterror. 2009;7(4):413-27.
33. Metz SW, Pijlman GP. Arbovirus vaccines; opportunities for the baculovirusinsect cell expression system. J Invertebr Pathol. 2011;107(Suppl):S16-30.

34. Frierson JG. The yellow fever vaccine: a history. Yale J Biol Med. 2010; 83(2):77-85.

35. Camacho LA, Freire Mda S, Leal Mda L, Aguiar SG, Nascimento JP, Iguchi T, et al. Immunogenicity of WHO-17D and Brazilian 17DD yellow fever vaccines: a randomized trial. Rev Saude Publ. 2004;38(5):671-8.

36. Hombach J, Solomon T, Kurane I, Jacobson J, Wood D. Report on a WHO consultation on immunological endpoints for evaluation of new Japanese encephalitis vaccines, WHO, Geneva, 2-3 September, 2004. Vaccine. 2005; 23(45):5205-11.

37. Hoke CH, Nisalak A, Sangawhipa N, Jatanasen S, Laorakapongse T, Innis BL, et al. Protection against Japanese encephalitis by inactivated vaccines. New Engl J Med. 1988;319(10):608-14.

38. Amicizia D, Domnich A, Panatto D, Lai PL, Cristina ML, Avio U, Gasparini R. Epidemiology of tick-borne encephalitis (TBE) in Europe and its prevention by available vaccines. Hum Vacc Immunother. 2013;9(5):1163-71.

39. Ishikawa T, Yamanaka A, Konishi E. A review of successful flavivirus vaccines and the problems with those flaviviruses for which vaccines are not yet available. Vaccine. 2014;32(12):1326-37.

40. Petersen LR, Powers AM. Chikungunya: epidemiology. F1000Res. 2016;5:82.

41. Wang JL, Li TT, Huang SY, Cong W, Zhu XQ. Major parasitic diseases of poverty in mainland China: perspectives for better control. Infect Dis Poverty. 2016;5(1):67.

42. Caraballo H, King K. Emergency department management of mosquitoborne illness: malaria, dengue, and West Nile virus. Emerg Med Pract. 2014; 16(5):1-23. quiz 23-24.

43. Mackenzie JS, Williams DT. The zoonotic flaviviruses of southern, southeastern and eastern Asia, and Australasia: the potential for emergent viruses. Zoonoses Public HIth. 2009;56(6-7):338-56.

44. Zhong YB, Liu XQ, Deng YC, Xu PH, Zhong GR, Zhang W. First case of laboratory-confirmed Zika virus infection imported into China. Chinese Med J-peking. 2016;129(16):2013-4.

45. Tappe D, Rissland J, Gabriel M, Emmerich P, Gunther S, Held G, et al. First case of laboratory-confirmed Zika virus infection imported into Europe, November 2013. Euro Surveill. 2014;19(4).

\section{Submit your next manuscript to BioMed Central and we will help you at every step:}

- We accept pre-submission inquiries

- Our selector tool helps you to find the most relevant journal

- We provide round the clock customer support

- Convenient online submission

- Thorough peer review

- Inclusion in PubMed and all major indexing services

- Maximum visibility for your research

Submit your manuscript at www.biomedcentral.com/submit 